\title{
Using a discrete thermal model to obtain a linear frequency ramping in a frequency-modulated continuous-wave system
}

\author{
Daniel Nordin \\ Kalevi Hyyppä \\ Luleå University of Technology \\ EISLAB \\ SE-971 87, Luleå, Sweden \\ E-mail: Daniel.Nordin@sm.luth.se
}

\begin{abstract}
The lasing wavelength of a single-section distributed feedback (DFB) laser diode can be modulated by modulating the drive current. This makes it possible to utilize the DFB laser diode in a frequencymodulated continuous wave (FMCW) range and velocity measuring system. In FMCW, the frequency of the laser is ramped, and the frequency difference between the reflected wave and a local-oscillator wave is monitored. For maximum performance the frequency ramping should be linear. Due to thermal phenomena, a linear ramping of the current seldom results in a linear ramping of the optical frequency. We have derived a discrete thermal model, using resistors and capacitors, of our laser module. The thermal model was then used as a starting point to model the frequency behavior of the laser and to derive modulation currents that resulted in a linear frequency ramping at some different modulation frequencies. (๑) 2005 Society of Photo-Optical Instrumentation Engineers. [DOI: 10.1117/1.1949205]
\end{abstract}

Subject terms: lidar; fiber optic sensors; thermal effects; tunable lasers.

Paper 040675R received Sep. 15, 2004; revised manuscript received Jan. 19, 2005; accepted for publication Jan. 31, 2005; published online Jul. 7, 2005.

\section{Introduction}

Frequency-modulated continuous wave (FMCW) is a range and radial-velocity measuring technique well known from radar applications. The reflected part of an outgoing frequency-modulated wave (w1) is mixed with a localoscillator wave (w2), and as a result the frequency difference between the two signals can be obtained. If the frequency modulation waveform is triangular, both radial velocity and range of the target can be determined from the frequency difference between w1 and w2, usually referred to as the beat frequency.

By using a tunable laser as the source, with the light frequency as the carrier frequency and a photodiode as detector, the beat frequency can be extracted directly from the photodiode current. One suitable type of light source is the distributed feedback (DFB) laser diode. By modulating the current to the DFB laser, the optical frequency can be modulated. Unfortunately, a linear ramping of the current seldom results in a linear ramping of the optical frequency. One solution to this problem is to use an optical reference path and divide the beat frequency from the reference path by the one obtained from the target. ${ }^{1}$ A second approach is to use an electrical feedback path to control the current ramp. ${ }^{2}$ The nonlinear frequency response of a multisection DFB was also investigated and compensated for in Ref. 3.

Our approach, presented in this paper, is to derive a model of the frequency behavior and use that model to find a suitable modulation current. This approach can be combined with the ones in Refs. 1 and 2.

\section{Modulation and Detection}

The simplest modulation scheme consists of a sawtooth waveform, which only allows range measurements to a stationary target. A more sophisticate FMCW scheme is displayed in Fig. 1. It has the advantage that both range $R$ and radial velocity can be calculated from two measured beat frequencies $f_{1}$ and $f_{2}$. If we assume that the range and velocity of the target are constant during one modulation period, $f_{1}$ and $f_{2}$ can be expressed as ${ }^{4}$

$f_{1}=\left|f_{\mathrm{R}}-f_{\mathrm{D}}\right|$,

$f_{2}=\left|-f_{\mathrm{R}}-f_{\mathrm{D}}\right|=\left|f_{\mathrm{R}}+f_{\mathrm{D}}\right|$,

where $f_{\mathrm{R}}$ is the magnitude of the frequency difference due to the range and $f_{\mathrm{D}}$ is the Doppler shift. If $\left|f_{\mathrm{D}}\right|<f_{\mathrm{R}}$, the frequency $f_{\mathrm{R}}$ can be expressed as

$f_{\mathrm{R}}=\frac{f_{1}+f_{2}}{2}=\frac{4 R \Delta f}{c T_{\mathrm{mod}}}$

and $f_{\mathrm{D}}$ as

$f_{\mathrm{D}}=\frac{f_{2}-f_{1}}{2}=\frac{2 v_{t}}{\lambda}$

where $\lambda$ is the optical wavelength. The range $R$ can be calculated from $f_{\mathrm{R}}$, and the radial velocity $v_{t}$ can be calculated from $f_{\mathrm{D}}$. If the distance between source and target decreases, the sign of $f_{\mathrm{D}}$, and hence $v_{t}$, will be positive. ${ }^{5}$ For maximum performance, the frequency ramping should be as linear as possible. If the frequency ramping is nonlinear, the beat frequency will vary during the ramping, and

0091-3286/2005/\$22.00 @ 2005 SPIE 

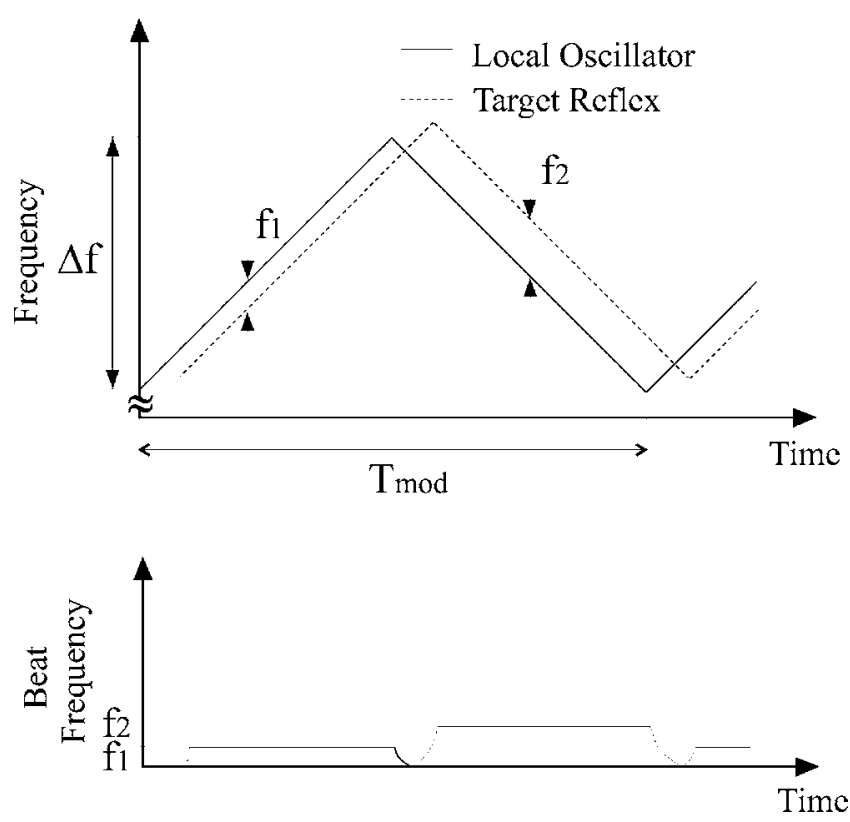

Fig. 1 Triangular modulation scheme, where the solid line corresponds to the local-oscillator wave and the dashed line to the reflected wave. The lower part shows the corresponding beat frequencies.

this smearing will make the beat frequency harder to detect. See Sec. 6 for a more detailed explanation of how a nonlinear frequency ramping affects the beat frequency.

When the reflected wave (w1) and the local-oscillator wave (w2) are mixed in a photodiode, the photodiode current will be proportional to the squared sum of the electrical fields of $\mathrm{w} 1$ and $\mathrm{w} 2$. Since the photodiode has limited bandwidth, the optical frequency components will be filtered out, and only the beat frequency component, corresponding to the frequency difference between w1 and w2, will be observable in the photodiode current.

\section{Laser Sources, System, and Performance}

The most common type of lasers used in FMCW systems today are diode lasers of distributed feedback (DFB) or distributed Bragg reflector (DBR) type, $\mathrm{CO}_{2}$ or $\mathrm{Nd}: \mathrm{YAG}$. The ideal source should operate in single mode and have long coherence length and high output power. Submicrometer resolution over shorter distances has been reported by some authors, ${ }^{6}$ and others have achieved a maximum range

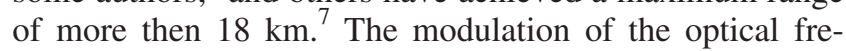
quency can often be realized by modulating a current to the modulation section of the laser. Some lasers have special connections for this purpose, but other lasers lacking the modulation section can often be modulated by adding a modulation to the regular drive current. Our laser is a single-section DFB, and hence current modulation is added to the drive current.

Changes in the drive current affect the lasing wavelength by two different mechanisms, the plasma effect and thermal effects. The plasma effect refers to how changes in the current density affect the refractive index of the material, which in turn affects the wavelength. Changes in temperature affect both the optical gain profile of the material and its dimensions, both of which in turn affect the lasing

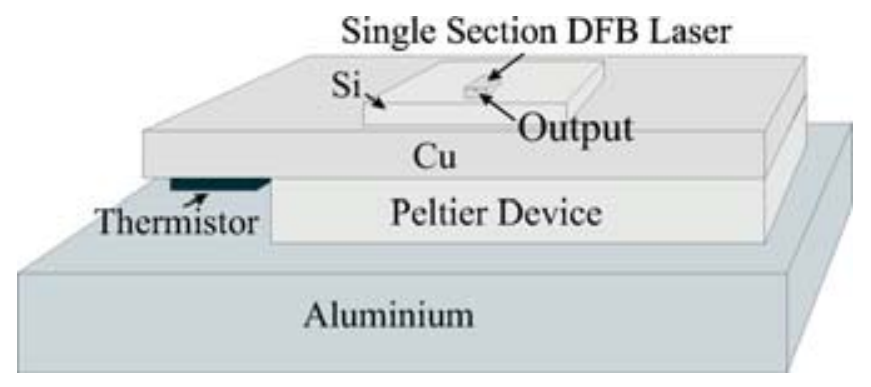

Fig. 2 A simple cross-section sketch of the laser module's different thermal parts. The parts are not drawn to scale.

wavelength. The time constant associated with the plasma effect has been reported to be in the nanosecond region. ${ }^{8}$

\section{Modeling the Frequency Behavior}

At modulation periods much longer then the time constant associated with the plasma effects, the thermal effects will dominate the dynamics of the frequency behavior. For this reason the thermal behavior of our laser module was investigated in Ref. 9. The idea is to obtain a simple model of the temperature dynamics, and hence the frequency behavior of the laser module with respect to changes in the drive current.

A sketch of our laser module's different thermal parts is shown in Fig. 2. The DFB laser chip is mounted on a silicon plate, and the silicon plate is mounted on a copper plate. The copper plate is mounted on a Peltier element, and the other side of the Peltier element is connected to a large aluminum block. As a starting point for our model we calculated a thermal capacitance and resistance for each thermal part. The thermal resistance of a plate can be calculated approximately from

$R_{\mathrm{th}}=\frac{1}{k}\left(\frac{\text { thickness }}{\text { area }}\right)$,

where $k$ is the thermal conductivity. The thermal conductivity depends on the material, temperature, geometry, and other environmental factors, but in our calculations we have treated $k$ as a constant. The thermal capacitance determines the material's ability to store energy in the form of heat and can be calculated approximately from

$C_{\mathrm{th}}=C_{\mathrm{s}} m$,

where $C_{\mathrm{s}}$ is the specific heat and $m$ the mass of the body.

To calculate the thermal resistance of the laser body from the active channel to the surface, we assumed that the whole body could be modeled as an indium phosphide (InP) cylinder with radius $r_{2}$. The active channel was modeled as a cylinder in the middle of the laser body with radius $r_{1}$. A cylinder with radius $r$ has a lateral surface area equal to $2 \pi l r$, where $l$ in this case corresponds to the length of our laser chip. By integrating over the cylinder we obtained the following equation for the thermal resistance from the active channel to the outside of the laser body:

$R_{\text {laser }}=\frac{1}{k} \int_{r_{1}}^{r_{2}} \frac{1}{2 \pi l r} \mathrm{~d} r=\frac{1}{2 \pi k l} \ln \left(\frac{r_{2}}{r_{1}}\right)$. 


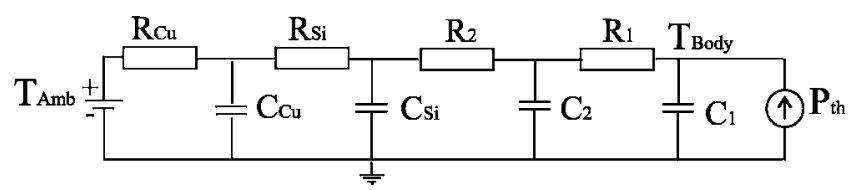

Fig. 3 Model used to simulate the thermal behavior of the laser. The components $R_{1}, R_{2}, C_{1}$, and $C_{2}$ model the laser body. The silicon and copper plates are modeled by $R_{\mathrm{Si}}, C_{\mathrm{Si}}, R_{\mathrm{Cu}}$, and $C_{\mathrm{Cu}}$. In the model, voltage corresponds to temperature and current corresponds to power.

The measurements reported in Ref. 9 showed that two thermal time constants had the largest influence on the frequency behavior. The shorter one was around $12.5 \mu \mathrm{s}$, and the longer one around $320 \mu \mathrm{s}$. The time constant for the active region was calculated as 18 ps with our model. This is too small to be measurable with our equipment. The FEMLAB simulation, also reported in Ref. 9, of the laser body indicated that $12.5 \mu \mathrm{s}$ is of the same order of magnitude as the time it takes to heat the Bragg region of the laser, while $320 \mu \mathrm{s}$ is of the same order of magnitude as the calculated time constant of the whole laser chip. The calculated thermal components and the measured time constants became the starting point of our model as displayed in Fig. 3. In the model, voltage corresponds to temperature and current corresponds to power. To take account of both the $12.5-\mu$ s and the $320-\mu$ s measured time constants, we used four components, $R_{1}, R_{2}, C_{1}$, and $C_{2}$, to model the laser body. The silicon plate is modeled by $R_{\mathrm{Si}}$ and $C_{\mathrm{Si}}$, and the copper plate by $R_{\mathrm{Cu}}$ and $C_{\mathrm{Cu}}$. Since the model is a very crude approximation with few components for each thermal block, it is not obvious if $R_{x}$, where $x \in(1,2, \mathrm{Cu}, \mathrm{Si})$, should be placed to the left or the right of $C_{x}$. Hence we tried both placements, using the measurement setup described in Sec. 6, and kept the one in the figure because it showed much better agreement between the measured and simulated results.

The input power $P_{\text {th }}$ represented by the current source can be calculated from $P_{\text {th }}=I_{\mathrm{D}} V_{\mathrm{D}}-\Phi$ where $I_{\mathrm{D}}$ is the laser drive current, $V_{\mathrm{D}}$ the laser voltage, and $\Phi$ the optical output power. The dc voltage source $T_{\mathrm{Amb}}$ corresponds to the external temperature. The idea is that if the laser temperature is directly proportional to the lasing wavelength, the voltage at the node $T_{\text {Body }}$ in Fig. 3 will be directly proportional to the laser's lasing wavelength. With an accurate model, it should be possible to replace the current source $P_{\text {th }}$ with a voltage source $T_{\text {th }}$, as in Fig. 4, and use a linearly ramping voltage $T_{\text {th }}$ to simulate a linear ramping of the temperature

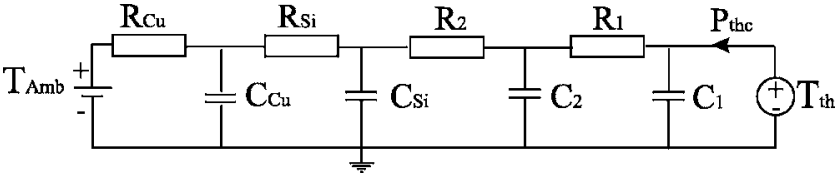

Fig. 4 Approach used to obtain a current waveform that results in a linear ramping of the temperature and hence frequency.

and wavelength. But what we really want is a linear ramping of the frequency. If the wavelength ramping is linear, it can be written as

$\lambda(t)=\lambda_{0}+\lambda_{\Delta} t$,

where $\lambda_{0}$ and $\lambda_{\Delta}$ are constants. For a linear frequency ramping $\mathrm{d} f / \mathrm{d} t$ should be constant. From $f=c / \lambda$ and Eq. (1) we can obtain

$\frac{\mathrm{d} f}{\mathrm{~d} t}=\frac{-c \lambda_{\Delta}}{\lambda_{0}^{2}+\lambda_{\Delta}^{2} t^{2}+2 \lambda_{0} \lambda_{\Delta} t}$,

which is approximately constant, since $\lambda_{0} \gg \lambda_{\Delta} t$. In our case $\lambda_{0} \approx 1,500,000 \lambda_{\Delta} t$. Hence, from now on, we make the assumption that a linear wavelength ramping results in a linear frequency ramping.

The high and low values of $T_{\mathrm{th}}$, which correspond to the start and end voltages of the ramp, used when simulating the circuit in Fig. 4, were obtained by simulating the circuit in Fig. 3 with the selected values of $I_{\mathrm{D}}$ and $V_{\mathrm{D}}$ and recording the high and low voltages at the node $T_{\text {Body }}$. From the current supplied by $T_{\text {th }}\left(P_{\text {thc }}\right.$ in Fig. 4), the compensated drive current to the laser can be derived using

$I_{\mathrm{Dc}}=\frac{P_{\mathrm{thc}}+\Phi}{V_{\mathrm{D}}}$.

When deriving the compensated drive current $I_{\mathrm{Dc}}$, the voltage-current and current-output-power relationships of the laser have to be taken into account. These relationships can often be modeled in the electrical circuit simulator used to simulate the thermal-wavelength model. With the current values used in our experiments, both the voltage-current and current-output-power relationships were approximately linear with $V_{\mathrm{D}}=1.45 \mathrm{~V}$ at $I_{\mathrm{D}}=59.2 \mathrm{~mA}, V_{\mathrm{D}}=1.55 \mathrm{~V}$ at $I_{\mathrm{D}}$ $=72.4 \mathrm{~mA}, \Phi \approx 7 \mathrm{~mW}$ at $I_{\mathrm{D}}=59.2 \mathrm{~mA}$, and $\Phi \approx 7.4 \mathrm{~mW}$ at $I_{\mathrm{D}}=72.4 \mathrm{~mA}$. Since the relationships were approximately linear, we used a triangular voltage and current source to allow for the effect of $V_{\mathrm{D}}$ and $\Phi$, where the voltage source representing $V_{\mathrm{D}}$ was ramped from 1.45 to $1.55 \mathrm{~V}$, and the

Table 1 The data for the different thermal parts and the resulting component values.

\begin{tabular}{ccccccccc}
\hline \hline & $\begin{array}{c}k \\
\text { Material }\end{array}$ & $\begin{array}{c}C_{\mathrm{s}} \\
(\mathrm{W} / \mathrm{m} \mathrm{K})\end{array}$ & $\begin{array}{c}\rho \\
(\mathrm{J} / \mathrm{kg} \mathrm{K})\end{array}$ & $\begin{array}{c}l \\
\left(\mathrm{~kg} / \mathrm{m}^{3}\right)\end{array}$ & $\begin{array}{c}h \\
(\mathrm{~mm})\end{array}$ & $\begin{array}{c}w \\
(\mathrm{~mm})\end{array}$ & $\begin{array}{c}R \\
(\mathrm{~mm})\end{array}$ & $\begin{array}{c}C \\
(\mathrm{~K} / \mathrm{W})\end{array}$ \\
\hline $\mathrm{InP}$ (body) & 68 & 310 & 4810 & 0.6 & 0.2 & 0.1 & 16.65 & $17.8 \times 10^{-6}$ \\
$\mathrm{Si}$ & 141.2 & 700 & 2330 & 11 & 10 & 0.5 & 0.032 & 0.09 \\
$\mathrm{Cu}$ & 386 & 385 & 8960 & 20 & 18 & 2 & 0.014 & 2.484 \\
\hline \hline
\end{tabular}




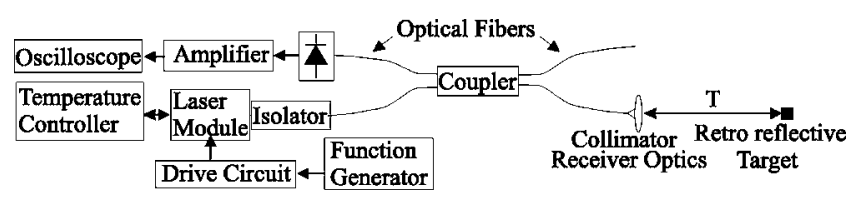

Fig. 5 The measurement system used to monitor the beatfrequency behavior resulting from the optical round trip delay $T$.

current source representing $\Phi$ from 7.0 to $7.4 \mathrm{~mA}$. In our case the drive circuit for the laser, which converts an input voltage to a drive current, also exhibited some nonlinearities that had to be compensated for.

The calculated component values of the whole laser body, $R_{\text {InP-Body }}$ and $C_{\text {InP-Body }}$, were divided between $R_{1}, R_{2}$, $C_{1}$, and $C_{1}$ according to $R_{2}=R_{\text {InP-Body }}-6 \Omega, C_{2}=C_{\text {InP-Body }}$ $-2 \mu \mathrm{F}, R_{1}=6 \Omega$, and $C_{2}=2 \mu \mathrm{F}$. These values, and the calculated component values for the $\mathrm{Si}$ and $\mathrm{Cu}$ plates in Table 1 , were used as the initial values in our model.

\section{Measurements}

Figure 5 displays the measurement system. An arbitrarywaveform function generator was used to generate the voltage waveforms. The laser drive circuit then converted this voltage into a current. The beat frequency $f_{\mathrm{b}}$, resulting from the optical round-trip delay $T$, as a function of time was monitored with an oscilloscope. In the experiments reported in this section $T$ was equal to $2.7 \mathrm{~ns}$. We also tested with $T=7.1 \mathrm{~ns}$ and $T=1.1 \mathrm{~ns}$, and the linearization improvements were similar for all three values of $T$.

Figure 6 shows the oscilloscope trace obtained with a linear current ramp from 59.2 to $72.4 \mathrm{~mA}$ and with $T_{\bmod }$ $=2 \mathrm{~ms}$. As can be seen in the figure, $f_{\mathrm{b}}$ is not constant during the ramp, and there is always a delay at the beginning of the ramp before the first solid beat-frequency period can be measured. In this case, the linear current ramping resulted in a nonlinear frequency ramping, which caused $f_{\mathrm{b}}$ to increase during the ramp. Figure 7 shows a crude theoretical example of the current, frequency, and beatfrequency behavior for a case like this. When the current is increased, we expect the lasing wavelength to increase and the frequency to decrease. If $\mathrm{d} f / \mathrm{d} t$ is approximately constant during $T$, then $f_{\mathrm{b}} / T \approx \mathrm{d} f / \mathrm{d} t$ and $f_{\mathrm{b}} \approx T \mathrm{~d} f / \mathrm{d} t$. If the lasing frequency $f(t) \approx f_{0}+A e^{t / \tau}$, where $f_{0}$ is the frequency at the turning point of the ramp and $A$ is positive or negative according as the frequency increases or decreases, then $f_{\mathrm{b}}(t) \approx T f^{\prime}(t)=(T A / \tau) e^{t / \tau}$. Since $f_{\mathrm{b}}^{\prime}(t)=\left(T A / \tau^{2}\right) e^{t / \tau}$ has the

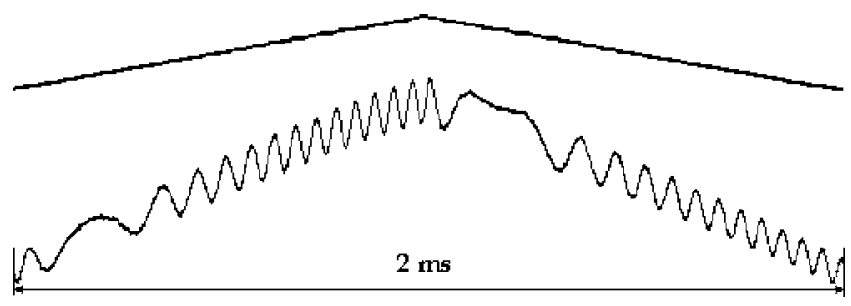

Fig. 6 The lower oscilloscope trace shows the beat frequency resulting from a linear current ramping. The upper trace shows the input voltage to the drive circuit.

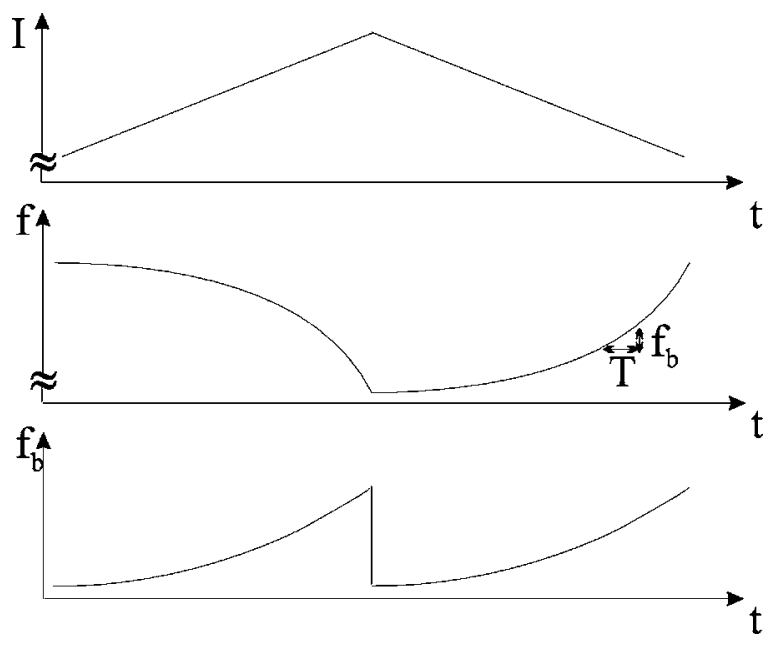

Fig. 7 Crude theoretical example of a linear current ramping that results in a nonlinear frequency ramping, which causes $f_{\mathrm{b}}$ to increase during both the up and down ramping of the current. If $\mathrm{d} f / \mathrm{d} t$ is approximately constant during $T$, then $f_{\mathrm{b}} / T \approx \mathrm{d} f / \mathrm{d} t$.

same sign as $f_{\mathrm{b}}(t)$, we expect $\left|f_{\mathrm{b}}\right|$, and hence the measured $f_{\mathrm{b}}$, to increase on both the up and the down slope of the current ramp.

The compensated modulation current obtained with our initial values for $R_{1}, C_{1}, R_{2}$, and $C_{2}$ resulted in a noticeable improvement over a linear ramping, but further optimization of the model was still needed. After some testing we ended up with $R_{2}=14 \Omega, C_{2}=45 \mu \mathrm{F}, R_{1}=2 \Omega$, and $C_{1}$ $=10 \mu \mathrm{F}$. The resulting modulation current at $T_{\bmod }=2 \mathrm{~ms}$ is displayed in Fig. 8, and Fig. 9 displays the oscilloscope view.

As a comparison we have plotted $f_{\mathrm{b}}$ versus time for both the regular current ramp and the compensated one in Fig. 10. When measuring a beat frequency period, the uncertainty on each period measurement was around $1.5 \mu \mathrm{s}$. This uncertainty, or noise, remained constant for all the different values of $T_{\text {mod }}$ that we tried, and as a result, the measured $f_{\mathrm{b}}$ values became more noisy at shorter beat-frequency periods.

The approach was tested for $T_{\mathrm{mod}}=8 \mathrm{~ms}, T_{\mathrm{mod}}=4 \mathrm{~ms}$, and $T_{\bmod }=1 \mathrm{~ms}$. In each of these cases we obtained a very good linearization of the frequency modulation, similar to what we did for $T_{\text {mod }}=2 \mathrm{~ms}$. At $T_{\text {mod }}=0.5 \mathrm{~ms}$ the result was somewhat worse than at lower modulation frequencies, but

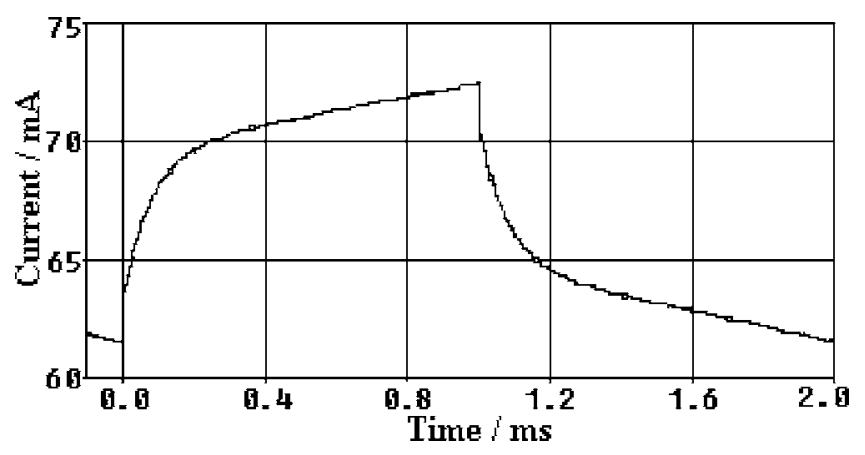

Fig. 8 The simulated compensation current. 


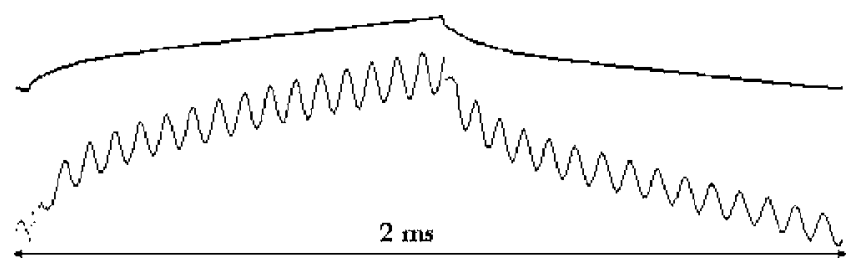

Fig. 9 The lower oscilloscope trace shows the beat frequency resulting from the compensated current ramping. The upper trace shows the input voltage to the drive circuit.

still much improved over linear current ramping, as can be seen in Fig. 11. We did not perform any measurements at higher modulation frequencies, since the drive circuit was not fast enough to deliver undistorted current waveforms.

We also tested with two different current amplitudes and at different operating current levels, and we obtained similar improvements to the one shown in Fig. 10. As long as the laser current did not drop too close to threshold, or exceed that of the drive circuit's maximum allowed laser current, we could not observe any dependence on $\mathrm{d} f / \mathrm{d} t$ and operating current. The model as such has no temperature limits, but if the laser is heated too much, it will not function properly. When the laser was operated in its intended temperature range, we could observe a slight temperature dependence on $\mathrm{d} f / \mathrm{d} t$. If the temperature was lowered by 5 $\mathrm{K}$ by using the Peltier device shown in Fig. 2, the mean value of $f_{\mathrm{b}}$ was lowered by approximately $5 \%$.

\section{Discussion}

The model is not perfect, and there is room for improvement. More components could be added to account for more time constants, and more time could be spent trying to find better component values. In all of our experiments there is a delay at the start of the ramp before the first period can be observed. This is expected, because at the turning point of the $\mathrm{ramp} \mathrm{d} I / \mathrm{d} t \rightarrow \infty$ and $f_{\mathrm{b}} \rightarrow 0$. In this

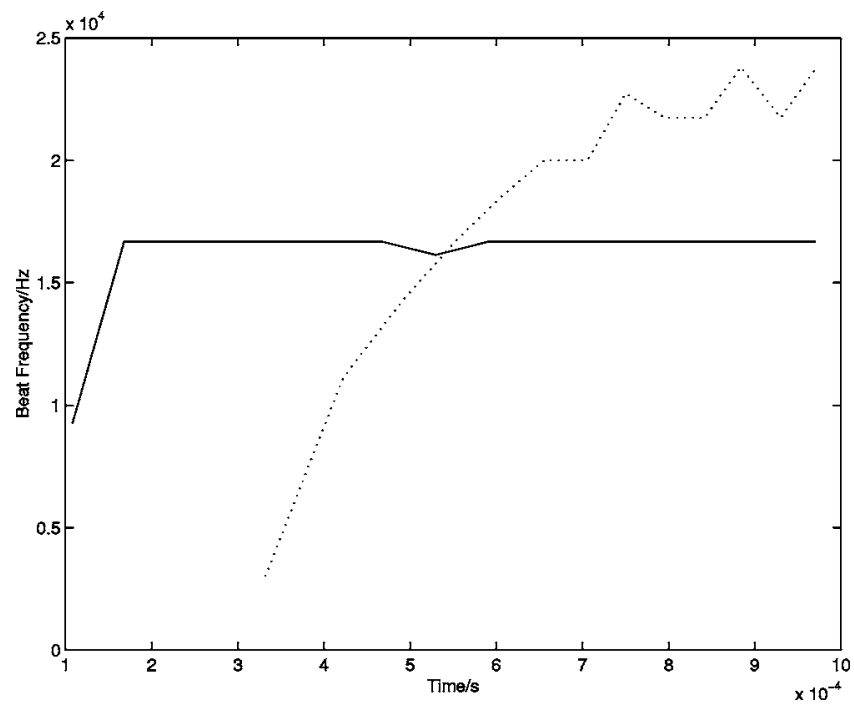

Fig. 10 The behavior of the beat frequency during the ramping of the current for $T_{\text {mod }}=2 \mathrm{~ms}$. The dotted line is the result of a linear current ramping, while the solid line is the result obtained with the compensated modulation current.

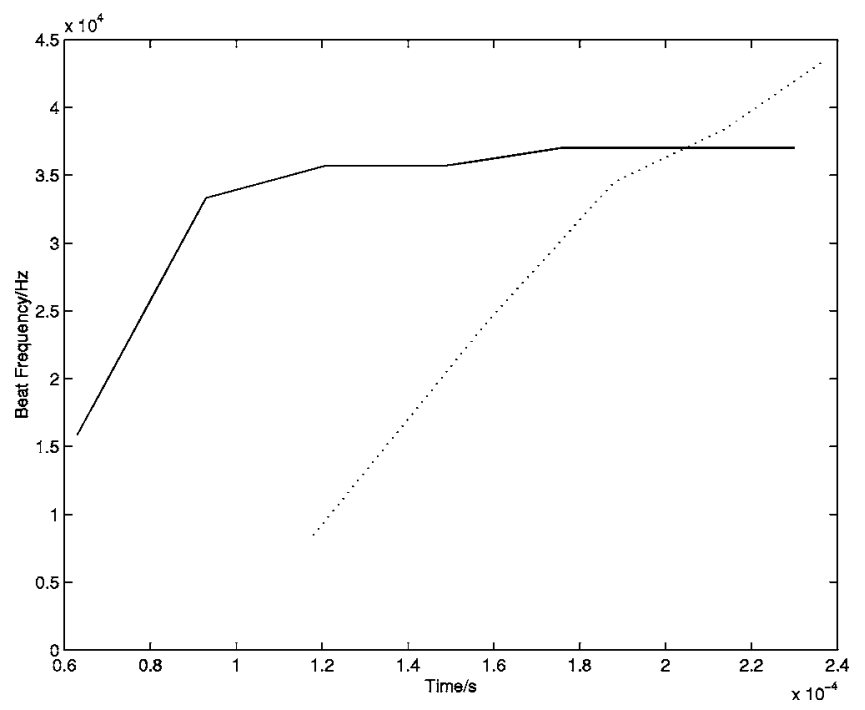

Fig. 11 The behavior of the beat frequency during the ramping of the current for $T_{\text {mod }}=0.5 \mathrm{~ms}$. The dotted line is the result of a linear current ramping, while the solid line is the result obtained with the compensated modulation current.

region the short time constants that are not included in our model, such as the ones associated with the plasma effect and the heating of the active region of the laser, will also have a larger influence on the beat frequency.

The $R C$ time constants that worked well in our model are not exactly the same as the 12.5 and $320 \mu$ s measured in Ref. 9 but they are on the same order of magnitude. In Ref. 9, the time constants were obtained by applying a current step to the laser, assuming that, $f_{\mathrm{b}} \approx T \mathrm{~d} f / \mathrm{d} t$, and monitoring the beat frequency using the same measurement setup as in Fig. 5. It is also worth noting that the value of $C_{1}+C_{2}$ that gave the best linearization is larger then the calculated capacitance for the whole laser body. We only had access to one DFB laser diode, but we expect that the approach should work well for other similar DFB laser diode modules.

\section{Conclusions}

Starting with a thermal analysis of our laser module, a simple discrete module for the temperature behavior was derived. By assuming that the lasing frequency is directly proportional to the temperature, we used the model to obtain modulation currents that resulted in a linear ramping of the optical frequency. The approach was tested with some different modulation frequencies and current amplitudes, with good result. Even though the result was not perfect at higher modulation frequencies, the linearity of the frequency sweep was noticeably improved compared to a triangular current ramping.

\section{References}

1. A. Dieckmann and M.-C. Amann, "Phase-noise-limited accuracy of distance measurements in a frequency-modulated continuous-wave LIDAR with a tunable twin-guide laser diode," Opt. Eng. 34, 896903 (1995).

2. I. Koichi, W. Lu-Tang, and H. Ken-ichi, "Linearizing optical frequency-sweep of a laser diode for FMCW reflectometry," J. Lightwave Technol. 14(2), 173-178 (1996).

3. C. J. Karlsson and F. A. Olsson, "Linearization of the frequency 
Nordin and Hyyppä: Using a discrete thermal model...

sweep of a frequency-modulated continuous-wave semiconductor laser radar and the resulting ranging performance," Appl. Opt. 38(15), 3376-3386 (1999).

4. D. Nordin and K. Hyyppä, "Advantages of a new modulation scheme in an optical self mixing FMCW system," Opt. Eng. 41(5), 11281133 (2002).

5. K. J. Gåsvik, Optical Metrology, 2nd ed., pp. 43-44, Wiley (1995).

6. E. C. Burrows and K.-Y Liou, "High resolution laser LIDAR utilising two-section distributed feedback semiconductor laser as a coherent source," Electron. Lett. 26(9), 577-579 (1990).

7. K. Nakamura, T. Hara, M. Yoshida, T. Miyahara, and H. Ito, "Optical frequency domain ranging by a frequency-shifted feedback laser,"
IEEE J. Ouantum Electron. 36(3), 305-316 (2000).

8. F. Delorme, P. Gambini, M. Puleo, and S. Slempkes, "Fast tunable $1.5 \mu \mathrm{m}$ distributed Bragg reflector laser for optical switching applications," Electron. Lett. 29, 41-43 (1993).

9. D. Nordin and K. Hyyppä, "Finding the thermal time constants of a DFB laser module used in a FMCW ranging system," in Proc. ODIMAP IV 4th Topical Meeting on Optoelectronic Distance Displacement Measurement and Applications, pp. 201-206, Infotech Oulu (2004).

Biographies and photographs of authors not available. 